\title{
Aprendizaje Basado en Proyectos: Explorando la caracterización personal del profesor de matemáticas
}

\author{
Project Based Learning: \\ Exploring the Mathematics Teacher's personal characterization
}

\author{
Brenda Rosales-Ángeles ${ }^{1}$ \\ Eric Flores-Medrano ${ }^{2}$ \\ Dinazar Isabel Escudero-Avila ${ }^{3}$
}

\begin{abstract}
Resumen
Al considerar un paradigma de acción en el aula, como lo es el Aprendizaje Basado en Proyectos (ABP), lo que es explorado y evidenciado desde el punto de vista académico, a veces está lejos de ser comprendido y retomado en el actuar diario del profesor. Perrenoud (2000) menciona que, dentro del método ABP, no hay un estándar, ya que todos tienen su propio entender y modos de ejecutarlo. Por esta razón, construimos una entrevista como instrumento para abordar la comprensión del profesor de matemáticas que generalmente trabaja con ABP y así elaboramos un instrumento con categorías y subcategorías basadas en distintos elementos, lo cual permite tener una mirada amplia y exhaustiva de la comprensión del profesor acerca del ABP.
\end{abstract}

Palabras clave: Aprendizaje Basado en Proyectos, Caracterización del ABP, Comprensión del profesor

\section{Resumo}

Ao considerar um paradigma de aula, como é a Aprendizagem Baseada em Projetos (ABP), aquilo que é explorado e evidenciado desde o ponto de vista acadêmico às vezes está longe de ser compreendido e retomado na ação cotidiana do professor. Perrenoud (2000) menciona que dentro do método ABP não há um padrão, já que todos têm seu próprio modo de entender e de executá-lo. Por isso, construímos uma entrevista como instrumento para acessar a compreensão do professor de matemática que geralmente trabalha com ABP e, assim, elaboramos um instrumento com categorias e subcategorias baseadas em distintos elementos, o qual nos permite ter uma visão ampla e exaustiva da compreensão do professor sobre o ABP.

Palavras-chave: Aprendizagem Baseada em Projetos; caracterização do ABP; entendimento do professor.

Submetido em: 05/11/2017 - Aceito em: 14/09/2018 - Publicado em: 16/09/2018

${ }^{1}$ Licenciado en Ingeniería en Alimentos por la Benemérita Universidad Autónoma de Puebla (México). Profesor de ciencias exactas. México.

${ }^{2}$ Doctor en Didáctica de las Matemáticas por la Universidad de Huelva (España), Maestro en Ciencias con especialidad en Matemática Educativa por el Centro de Investigación y de Estudios Avanzados (México), y Licenciado en Física y Matemáticas por el Instituto Politécnico Nacional (México). Profesor de la Licenciatura en Matemáticas y del Posgrado en Educación Matemática de la Benemérita Universidad Autónoma de Puebla. México.

${ }^{3}$ Doctor en Didáctica de las Matemáticas por la Universidad de Huelva (España), Maestro en Ciencias con especialidad en Matemática Educativa por el Centro de Investigación y de Estudios Avanzados (México), y Licenciado en Física y Matemáticas por el Instituto Politécnico Nacional (México). Profesora del Posgrado en Educación Matemática de la Benemérita Universidad Autónoma de Puebla México. 
DOI: https://doi.org/10.20396/zet.v26i3.8650908

\begin{abstract}
In considering a paradigm of action within the classroom, such as the Project Based Learning (PBL), which is explored and evidenced from the academic point of view, sometimes is far from being comprehended and taken up in the daily actions of the Professor. Perrenoud (2000) mentions that, within the PBL method, there is no a standard, since they all have their own ways of understanding and executing it. For this reason, we constructed an interview as a tool to address the understanding of the mathematics professor who usually works with the PBL and thus we produce an instrument with categories and subcategories based on various elements, this allows us to have a wide and comprehensive point of view of the understanding of the professor about the PBL.
\end{abstract}

Keywords: Project Based Learning, PBL characterization, comprehension of the professor.

\title{
Introducción
}

Los métodos de enseñanza-aprendizaje evolucionan para estar en concordancia con los procesos de cambio que se producen en lo social, cultural, económico, laboral y tecnológico, involucrando, por ejemplo, modificaciones en la metodología de trabajo que pueden afectar el rol del profesor y la forma en la que el alumno adquiere el conocimiento (Espinosa, Soler, Escoda, Puig y Ferrer, 2015). Para lograr que los estudiantes reconozcan la utilidad de los conceptos, las instituciones formativas han tenido que adquirir una mayor versatilidad y flexibilidad para adaptarse a las nuevas estructuras que se están desarrollando en el mundo (Tippelt y Lindemann, 2001), en lugar de basarse unicamente en la ejercitación y el manejo de algoritmos, que son poco identificables con problemas de la vida real (Batanero y Díaz, 2004; Aravena, 2002). El Aprendizaje Basado en Proyectos (ABP) es un ejemplo de este tipo de metodologia o estratégia didáctica. Según Moreti (2009), el uso de proyectos en la enseñanza de las matemáticas permite una ruptura con el contrato didáctico convencional, en la que los roles del estudiante y el maestro tienen características que limitan su actuar y su interacción.

El ABP es una metodologia didáctica que intenta reducir las limitaciones de métodos tradicionales, creando para el alumno situaciones más próximas a la realidad convirtiéndolo en el actor principal (Reverte, Gallego, Molina y Satorre, 2007). Galeana (2006) comenta que el ABP ofrece una gran oportunidad para el aprendizaje pues se forman equipos con personas de características diferentes que han de trabajar juntas para resolver un problema o trabajar un proyecto, preparando a los estudiantes de esta forma para un mundo diverso y global. Valero (2007), por su parte, habla de que este es un método que resulta motivador para los estudiantes y, por tanto, aumenta el rendimiento académico.

En resumen, el $\mathrm{ABP}$ es una metodología de enseñanza que ha sido ampliamente utilizada desde hace ya muchos años y que además ha sido conceptualizada, tal como lo mostraremos más adelante. Sin embargo, todas estas metodologías pueden sufrir modificaciones al ser aplicadas por los profesores dentro del aula por las concepciones sobre la enseñanza y aprendizaje de las matemáticas. Esto nos lleva a plantearnos cuáles son las ideas germinales y básicas del ABP que son consideradas por los profesores al aplicarlo en el aula. Por lo tanto, en este artículo nos planteamos el objetivo de mostrar el desarrollo de un instrumento que permite identificar la caracterización personal que hace el profesor de matemáticas acerca del ABP con la finalidad de poder compararla con los elementos que se 
plantean en la literatura especializada, el cual podría usarse en futuras investigaciones para plantear programas de formación docente.

\section{Marco Teórico}

Aunque en la literatura especializada existen diferentes conceptualizaciones del ABP, un punto en común que encontramos es que este comienza con la presentación de un producto o prototipo final. Su producción surge de la necesidad de resolver uno o más problemas, los cuales son reflejo de la vida real. El conocimiento adquirido y las destrezas empleadas durante la producción son muy importantes para el logro de los objetivos.

Dentro de la enseñanza de las matemáticas, el ABP ha dado resultados interesantes respecto a distintos temas. Por ejemplo, Batanero y Díaz (2004) presentan algunos ejemplos de proyectos que centran sobre aplicaciones de la estadística en contextos reales. Infieren que haciendo un cambio en el enfoque de las actividades curriculares con la incorporación de proyectos se trabajan distintos contenidos en lugar de concentrarse en un solo concepto, propiedad o capacidad.

En la construcción de modelos matemáticos, Aravena, Caamaño y Giménez (2008) muestran el diseño y aplicación de una propuesta didáctica en el tema de funciones que se apoya en la modelización a través de proyectos en grupo. Esto permite que los estudiantes observen el fenómeno desde distintas perspectivas al transitar por los diferentes sistemas de representación. En este trabajo se describen los elementos cognitivos, metacognitivos y transversales que se desarrollaron en los estudiantes desde la etapa de interpretación de datos y condiciones del problema (matematización, validación y verificación del modelo) hasta la comunicación con argumentos matemáticos ante sus compañeros y el profesor. Reportan que trabajar proyectos a través de la modelización "da significado a los conceptos y métodos matemáticos, apreciando la aplicabilidad de los conceptos, la utilidad de las representaciones gráficas y de la manipulación algebraica en la descripción matemática del fenómeno en estudio" (p.89).

En Morales y García (2015) se reporta la mejora que tienen los estudiantes en un curso de geometría y trigonometría respecto a su actitud ante las matemáticas en comparación con una clase tradicional, pues al trabajar con el ABP los alumnos son protagonistas de su propio aprendizaje y construyen su conocimiento motivados por el sentido de una aplicación real de las matemáticas.

Por otro lado, en Morales (2011) se hace una programación del curso de Álgebra que consta de un conjunto de proyectos y problemas entre los cuales se destaca una práctica de fin de curso en la que los alumnos apoyados con software libre matemático (OpenOffice Calc y wxMaxima) debían aplicar una gama de contenidos (áreas, perímetros, escalas, fórmulas, cambios de unidades de todo tipo, entre otros) para resolver el proyecto en cuestión. Con aquella experiencia se pudo comprobar que el alumno que experimenta el ABP "pasa de sentir desorientación durante las primeras sesiones, a sorpresa y admiración por la nueva 
visión global del alcance de las matemáticas y adquiere confianza en sus propias posibilidades como expertos en el área" (Morales, 2011, p.11).

En Benjumeda, Romero y López (2015) se pone de manifiesto el potencial de esta metodología para promover los procesos de alfabetización matemática, fundamentalmente los relacionados con la aplicación de conocimiento y modelización matemática. Pues la mayoría de los estudiantes reconocen la utilidad de los aprendizajes adquiridos. Pero los autores también manifiestan las dificultades y deficiencias del ABP en la forma en la que se gestionó el Proyecto y en ese grupo particular.

De manera general, Anijovich y Mora (2010) proponen una clasificación donde se habla de 4 tipos de proyectos: "1. De producto: Realizar algo en concreto [por ejemplo un prototipo de mejora para un artefacto]; 2. De resolución de Problemas: Involucra el Análisis de la situación y [propuesta de] una o varias soluciones; 3. De investigación: Se propone profundizar conocimientos [sobre un tema concreto], y 4. Elaboración de propuestas: Implica la puesta en marcha y el uso de un procedimiento" (p. 95).

Para tener éxito en el ABP es necesario "un diseño instruccional definido, definición de roles y fundamentos de diseño de proyectos" (Galeana, 2006, p.1). Además, el tipo de estrategia que se diseñe y las prácticas de enseñanza que le acompañen dependerán de las características de cada disciplina y la idea que cada docente tiene sobre qué es el conocimiento y cómo se accede a este (Anijovich y Mora, 2010). La implementación de la metodología dependerá en gran medida de las características que el profesor atribuye al método y de los actos que él proponga en su gestión.

\section{Metodología}

Como mencionamos anteriormente, nuestro interés es construir un instrumento que permita describir la caracterización personal de profesores de matemáticas sobre el ABP.

Para realizar esta construcción se utilizó la perspectiva metodológica top-down y bottom-up (Niss, 2006), que nos permitió un doble acercamiento para la caracterización del $\mathrm{ABP}$, teórica y personal. La Figura 1 muestra esquemáticamente el diseño de la investigación y cómo cada parte nos permitió llegar el objetivo final. 
DOI: https://doi.org/10.20396/zet.v26i3.8650908

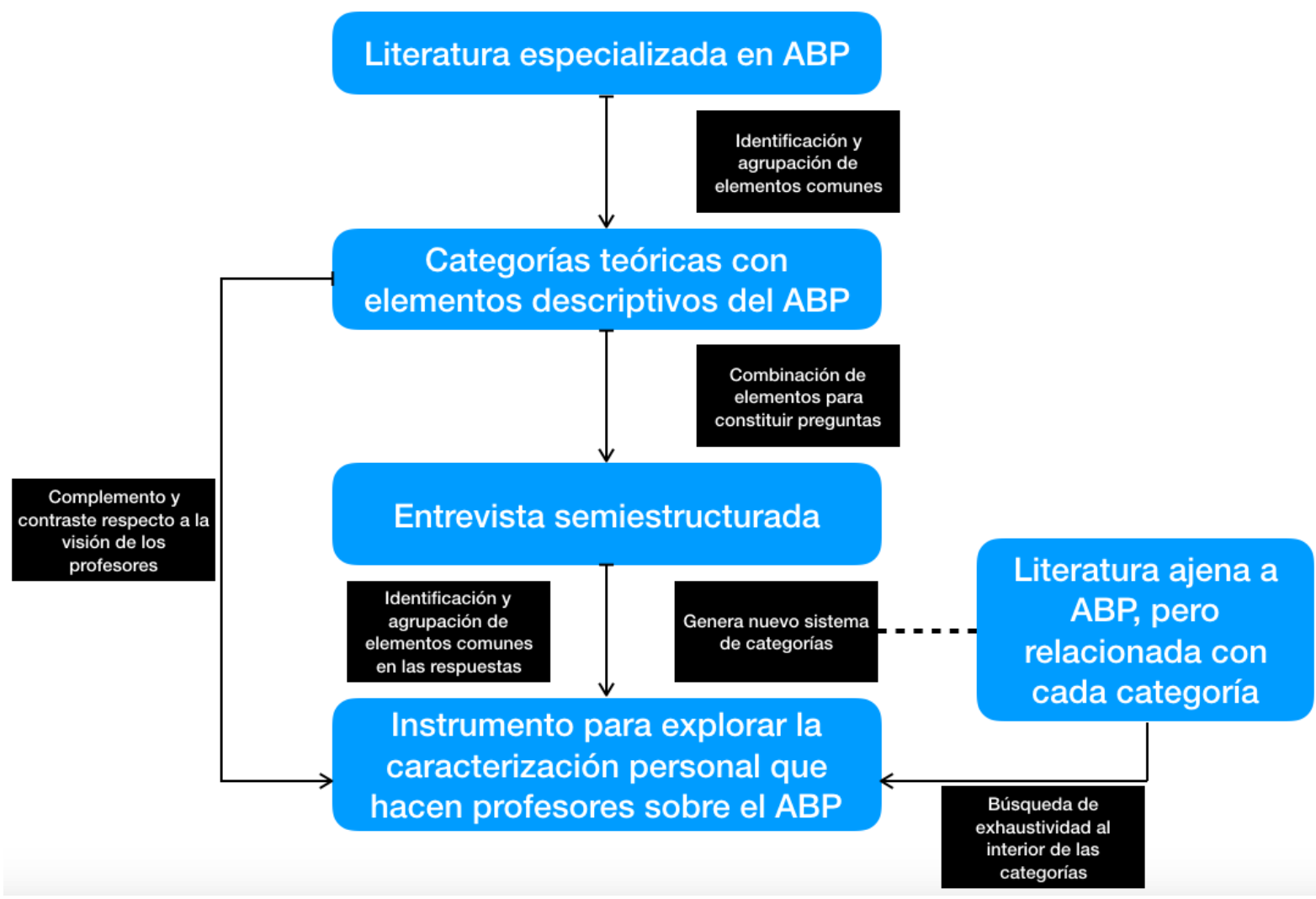

Figura 1. Diseño de la investigación y papel de las técnicas empleadas

A continuación, se describirán ambos acercamientos, así como la forma en la que se estructuró y diseñó la entrevista semiestructurada. Mencionaremos, también, las características de los informantes de este estudio.

\section{Acercamiento Top- Down}

En esta primera aproximación se busca información para obtener una idea general que pueda fungir como esquema formal (Nestingen, 2002).

En nuestra investigación se hizo un análisis de literatura de investigación especializada en el ABP que nos permitió construir una primera caracterización teórica de esa metodología. Este se construyó en dos fases, la primera enfocada en la literatura referente a las definiciones del ABP, su implementación en el mundo, en la educación matemática y sobre las concepciones de los profesores sobre el ABP (e.g. Perrenoud, 2000; Thomas, 2000), la cual organizamos de la siguiente forma:

\section{Propiedades inherentes al Aprendizaje Basado en Proyectos}

1P. Se utilizan problemas reales

2P. El poder del profesor está limitado y se lo transfiere a los alumnos, pero debe de conducirlos a tomar decisiones y hacer juicios basados en hechos.

3P. Habitualmente se trabaja en grupos de 6 a 8 integrantes. Para una correcta organización suele quedar al frente un responsable y se espera que todos los integrantes del equipo 
DOI: https://doi.org/10.20396/zet.v26i3.8650908

colaboren en cada una de las actividades.

4P. La longitud y la complejidad del proyecto son elegidas por el tutor de acuerdo a los objetivos que persigue.

5P. Está ligado a un aprendizaje previo y a un nuevo conocimiento de la misma o de distinta disciplina.

6P. Además de lograr los objetivos del proyecto (tener éxito) se debe conseguir un aprendizaje.

7P. Es importante realizar evaluaciones constantemente.

\section{Beneficios de $A B P$}

1B. Como la experiencia que se vive es auténtica los alumnos vuelven a ella de manera reflexiva y analítica y los nuevos saberes se fijan.

2B. Se contribuye al desarrollo del pensamiento reflexivo.

3B. El alumno practica la autoevaluación; está consciente no sólo de lo que sabe sino de su capacidad para utilizarlo en una situación y la posibilidad y vías para desarrollar esta capacidad. Cuando los conocimientos que posee son escasos y no es posible remediar esta carencia, por lo menos puede percatarse de la necesidad de formación. Esto le permite al estudiante identificar sus fortalezas y debilidades.

4B. Los estudiantes entran en contacto con la sociedad y descubren nuevas facetas de la cultura.

5B. Se desarrollan competencias como trabajar en equipo, escuchar, formular propuestas e hipótesis, tomar decisiones y cumplirlas, negociar, dirigir proyectos (si el alumno asume ese rol), coordinar tareas, planear y respetar cronogramas.

6B. Se estimula la creatividad y responsabilidad de los participantes.

7B. Los proyectos que se inician a principio del ciclo escolar evitan que se den por sentado roles para los distintos integrantes de acuerdo al conocimiento personal que el resto tiene de ellos.

\section{Dificultades de $A B P$}

1D. Los fallos pueden vivirse como fracaso y hacer sentir al alumno incompetente.

2D. Se puede renunciar por no poseer saberes o procedimientos o por no saber cómo adquirirlos a tiempo.

3D. Se corre el riesgo de que algunos alumnos asuman, sin estar convencidos, ideas colectivas que no comprendan o no compartan.

4D. Como a veces no se alcanzan todos los objetivos marcados en el programa, se genera la concepción de que este se debe de cambiar (Dificultad institucional). 
5D. Los diferentes actores del proceso de enseñanza y aprendizaje pueden tener la sensación de que no se adquirió el conocimiento suficiente al término del proyecto.

6D. Los alumnos pueden caer en el error de dividirse el trabajo en lugar de que todos participen en cada una de sus fases.

7D. En los primeros trabajos con proyectos se presenta un cierto nivel de resistencia por parte de los alumnos ya que tienen la tendencia a regresar a las metodologías tradicionales en las que suelen estar inmersos.

8D. Enfrenta al alumno a obstáculos que en ocasiones no puede/quiere superar de manera individual.

9D. Se requiere un cambio de aptitudes y actitudes de todos, y como el ABP se centra en el alumno, este debe poseer ciertas cualidades o estar dispuesto a desarrollarlas y sin ello no es posible tener éxito en el método.

Como mencionamos antes, estos elementos fueron extraídos de literatura específica sobre ABP. Algunas fuentes consideraban pertinente el uso de dicha metodología didáctica y otras fuentes la criticaban (motivo por el cual la organización se da en términos de beneficios, dificultades y propiedades inherentes, siendo neutras estas últimas). Dichos elementos son, en su mayoría, presentados de manera categórica, es por ello que requerimos de un segundo acercamiento para generar datos que nos permitieran reorganizar estas características como parte del pensamiento del profesorado acerca del ABP.

\section{Acercamiento Bottom-up}

En este acercamiento se analizaron las entrevistas realizadas a tres profesores de matemáticas que trabajan o han trabajado con ABP. Hablaremos en secciones posteriores sobre la construcción y análisis de estas entrevistas puesto que nos proporcionaron elementos indispensables para la refinación del instrumento de caracterización personal del profesor sobre el ABP.

Planteamos este segundo acercamiento para obtener, de manera indirecta, pero relacionada con su práctica real, el parecer de los profesores acerca de los elementos que hallamos en la literatura especializada. Esto nos permitió una reorganización de categorías y el planteamiento de una variedad de opciones de caracterización en cada una de estas.

\section{Informantes}

Los informantes fueron tres profesores de matemáticas mexicanos de distintas instituciones educativas mexicanas en las que se trabaja el ABP. Todos suelen utilizar el ABP para trabajar algunos temas de sus clases de matemáticas. El profesor PG es Ingeniero Civil, ha tomado distintos cursos de formación y actualmente se encuentra estudiando una maestría en Educación Matemática. Imparte las asignaturas de matemáticas, estadística, cálculo, física y alcantarillado (media superior y superior), cuenta con 4 años de experiencia como profesor. El profesor PA es Licenciado en Electrónica y tiene el grado de Maestro en Educación de Ciencias Exactas, actualmente imparte las asignaturas de matemáticas y física (Secundaria y 
bachillerato), cuenta con más de 16 años de experiencia. El profesor PE cuenta con licenciatura, maestría y doctorado en matemáticas, además un doctorado en Ingeniería Mecatrónica. Ha tomado cursos de capacitación y diplomados como instructor de programas de formación docente, desarrollo de competencias para profesores de bachillerato, de creatividad y de innovación educativa. Actualmente imparte las asignaturas de matemáticas (Universidad) y herramientas aprendizaje autónomo. Tiene más de 30 años de experiencia como docente.

Toda vez que no era nuestra intención generalizar sobre la concepción que los docentes de matemáticas tienen acerca del ABP, sino construir un instrumento, con base teórica y empírica, que permita analizar la variedad de caracterizaciones que un profesor pueda plantearse sobre el Aprendizaje Basado en Proyectos, los tres profesores entrevistados fueron suficientes para dicha finalidad.

\section{Construcción y validación de la entrevista como instrumento de recogida de información}

Una vez caracterizado el método de ABP y habiendo revisado publicaciones donde se muestran ejemplos de la aplicación de dicho método, elaboramos una entrevista semiestructurada (Blasco y Otero, 2008) y focalizada (Valles, 2002). El guion de la entrevista se elaboró a partir de la caracterización teórica del ABP mostrada anteriormente. Está dirigida a profesores que han trabajado o trabajan en sus aulas con el método de Aprendizaje Basado en Proyectos.

A continuación, presentaremos la estructura de la entrevista y ejemplificamos la forma en la que construimos las preguntas. A partir de la pregunta 4, se coloca uno o más códigos compuestos de un número y una letra entre paréntesis al finalizar el texto del cuestionamiento. Estos códigos se corresponden con la caracterización teórica presentada en el apartado anterior.

El primer bloque de la entrevista corresponde a los datos personales, formación y experiencia del docente.

El segundo bloque, correspondiente a las preguntas 1 a la 3 (Cuadro 1). Se centra en verificar si el entrevistado reconoce que aplica el ABP como una metodología en el aula y también buscamos tener un primer acercamiento general hacia el significado que le asocia a dicha metodología.

Cuadro 1. Segundo bloque de la entrevista

1. En este Instituto se aplica la metodología de Aprendizaje basado en proyectos ¿Qué ha escuchado acerca de este método?

2. ¿Lo ha utilizado?

3. ¿Podría definir en qué consiste?

El tercer bloque de preguntas, que va de la 4 a la 7 e incluye también a la 17 (Cuadro 
DOI: https://doi.org/10.20396/zet.v26i3.8650908

2) se enfoca en el diseño y elección del proyecto. Se deseaba identificar qué aspectos espera el profesor que tenga un proyecto para considerarlo como aplicable en su aula.

Cuadro 2. Tercer bloque de la entrevista

4.¿Tiene en mente algún proyecto con el que haya trabajado?, ¿en qué consistía? $(\mathrm{P} 1)(\mathrm{P} 4)(\mathrm{P} 6)$

5.¿Qué aspectos considera que favorecieron al proyecto? $(\mathrm{P} 5)(\mathrm{P} 4)(\mathrm{B} 1)$

6.¿Qué aspectos piensa que pudieron haberlo afectado o que pudiesen haber sido mejorados?(P4)(D2)(D7)(D9)

7.¿Cómo elige o diseña un proyecto?, ¿qué aspectos toma en cuenta?(P1)(P4)(P5)(P7)(B3)(B5)

17. ¿Existe algún o algunos temas matemáticos que no recomendaría trabajar con ABP? $(\mathrm{P} 1)(\mathrm{P} 4)(\mathrm{D} 1)(\mathrm{D} 2)(\mathrm{D} 4)$

El cuarto bloque comprende las preguntas 8 a la 11 (Cuadro 3) y se enfoca en la gestión del proyecto que involucra aspectos como su duración, la descripción de las actividades para estar en concordancia con los programas académicos y los roles $\mathrm{u}$ obligaciones de los actores involucrados.

Cuadro 3. Cuarto bloque de la entrevista

8.¿Cuánto es el tiempo máximo que le ha llevado estar trabajando con un proyecto? (P4)

9.¿Es posible controlar los avances que piden los programas oficiales? (D4)(D5)

10.¿Cuál es su papel o función como profesor al trabajar con proyectos? (P2)

11.¿Cuáles son las responsabilidades o funciones del alumno?

(P3)(B3)(B4)(B5)(B6)(D6)(D9)

En el quinto y último bloque de preguntas de la 12 a la 16 se aborda el tema de la evaluación, el cual se divide en dos partes en la primera (Cuadro 4A) nos referimos a la evaluación del estudiante y en la segunda (Cuadro 4B) hacemos referencia a la evaluación del ABP.

Evaluación del estudiante:

Cuadro 4A. Quinto bloque de la entrevista primera parte

12.¿Cómo es la actitud de los estudiantes cuando se enfrentan al ABP por primera vez?, ¿cambia en posteriores?, ¿de qué depende? $(14,21,22,23)$

13. ¿Qué aspectos toma en cuenta para la evaluación? (P6)(P7)(B3)(B5)(B6) 
DOI: https://doi.org/10.20396/zet.v26i3.8650908

Evaluación del ABP:

Cuadro 4B. Quinto bloque de la entrevista segunda parte

14. Con respecto a una clase tradicional, ¿se mejoran algunos aspectos al trabajar con proyectos? En los estudiantes, la clase o el conocimiento adquirido (B2)(B3)(B4)(B5)(B6)

15. ¿Qué ventajas hay de trabajar ABP? (B1)(B2)(B3)(B4)(B5)(B6)(B7)

16. ¿Qué riesgos se corren al trabajar con ABP? (D1)(D2)(D3)

Por otro lado, como ya lo hemos mencionado, cada pregunta de la entrevista tiene correspondencia con algunos elementos de la caracterización teórica del ABP que elaboramos. Esta correspondencia se relaciona con los elementos que esperábamos que se pusieran en juego en las probables respuestas, pero cuidamos que las preguntas quedaran lo suficientemente abiertas para evitar sesgos producto de su propia redacción/aplicación.

Para ilustrar la forma en la que construimos la entrevista tomamos como ejemplo la pregunta 4:

“¿Tiene en mente algún proyecto con el que haya trabajado?, ¿en qué consistía?”(1P)(4P)(6P)

Al preguntarle por la descripción de un proyecto, se esperaba que el profesor se refiriera de manera indirecta a las características que atribuye al ABP, tomando en cuenta, por lo menos, el tipo de problemas que emplearía (1P), la duración del proyecto (4P) y una abstracción de los objetivos que se planteaba (6P). Pensamos que estas características tienen una íntima relación con los elementos que constituyen la elección, diseño y planeación del proyecto en la asignatura.

Una vez elaborada la entrevista se realizó una triangulación de investigadores la cual tuvo por objetivo "detectar o minimizar los sesgos que introduce la propia persona del investigador" (Carrillo y Muñoz-Catalán, 2011, p.94) y consistió en compartir con otros investigadores expertos los resultados y análisis de los datos. Con esta validación pudimos comprobar que el instrumento tenía coherencia en cuanto a contenido, pertinencia y orden de las preguntas.

Con estas agrupaciones se desarrollaron categorías y subcategorías, por ejemplo, en la 4P: Sobre los aspectos que favorecen o afectan la incorporación del ABP como metodología de trabajo, los tres profesores entrevistados coincidieron en el elemento "Disponibilidad" de los estudiantes.

También ubicamos características expresas de un profesor que en la entrevista de otro profesor no aparecieron como en la Categoría 1: Sobre la elección del problema o temática, un solo entrevistado dio indicios de ubicar actividades sin contexto en $\mathrm{ABP}$, lo que nosotros después con el acercamiento Top- Down denominamos "Puro" 
Este instrumento consta de 6 elementos y cada uno de ellos se divide en subelementos, los cuales tienen la intención de recoger información de forma que se implique toda aquella característica, cualidad o actividad que sea definida por el profesor de matemáticas como propia del ABP. Dicho de otro modo, las categorías y subcategorías buscan cubrir un amplio espectro, lo cual permita realizar análisis de otros profesores y que sus caracterizaciones encuentren explicación a partir del instrumento que desarrollamos.

\section{Instrumento de categorización de elementos del ABP}

A continuación, presentamos los elementos que conforman el instrumento de caracterización del ABP, las subcategorías con su respectiva descripción así como algunos ejemplos representativos de lo que los profesores argumentaron en la entrevista sobre dichos elementos (Las respuestas completas de los profesores pueden consultarse en el Anexo 2), para lo cual utilizaremos el código PG, PA y PE para referirnos a los tres profesores respectivamente.

\section{Categoría 1: Sobre la elección del problema o temática}

Esta categoría sirve para analizar la naturaleza y fuente del (de los) situaciones(s)/problema(s) que utiliza el profesor cuando emplea el ABP como metodología de enseñanza. La subdividimos en cuatro subcategorías: reales, cuasireales, ficticios y puros.

Real: Este tipo de contextos responden a la idea de los profesores de apegarse a la matemática realista. Son sucesos cotidianos para el estudiante porque están dentro de su cultura y sociedad (Villa, Rojas y Cuartas, 2010). Por ejemplo:

PG: [Los estudiantes] desarrollan estudios estadísticos sobre algún tema: uso de dinero dentro de la escuela, redes sociales, preferencias sexuales.

Cuasi- Real: Son situaciones artificiales, sin impresiones sensoriales, imaginadas por el autor del problema y descritas completamente por el texto del ejercicio, es decir que no es necesaria información adicional (Skovsmose, 2001). Esta subcategoría la construimos teóricamente, ninguno de los informantes expresó utilizar problemas de esta naturaleza.

Ficticios: Se consideran dentro de esta categoría los contextos imaginativos, de fantasía, o circunstancias inusuales (Wiest, 2001). Esta subcategoría la construimos teóricamente, ninguno de los informantes expresó utilizar problemas de esta naturaleza.

Puro: Está dominado por la tradición de los ejercicios basados en la investigación de números o figuras geométricas (Skovsmose, 2001). Hace referencia a aquellos problemas que no son ni cotidianos ni conocidos (Falcao, 2014) que sólo se preocupan por el estudio y desarrollo de las estructuras matemáticas. Por ejemplo:

PA: [Analizamos] figuras geométricas sencillas, prismas, pirámides, poliedros.

\section{Categoría 2: Sobre el papel del Profesor}


DOI: https://doi.org/10.20396/zet.v26i3.8650908

En esta categoría se consideran las funciones que reconoce/evidencia el profesor antes, durante y después de la aplicación de un proceso de proyecto. Por esta razón, la categoría queda dividida en las subcategorías Diseña, Gestiona y Evalúa.

Diseña: En la literatura especializada que revisamos (e.g. Batanero y Díaz, 2004), la mayor parte de los proyectos son trabajados en educación superior y son los estudiantes quienes encargan del diseño del proyecto. Sin embargo, con base en los datos recogidos para esta investigación (en los que aparecen profesores de nivel básico, medio y superior) los profesores también suelen asumir un rol principal en la planificación del proyecto. Esta categoría recoge los elementos que el profesor reconozca/evidencie como su participación en el diseño del proyecto.

PE: [Para diseñar o elegir un proyecto] tienes que ubicarte en el contexto de los estudiantes, eso es esencial, tú no le puedes poner un problema que no sea de su contexto y de ahí partes, sobre todo lo que tú quieres enseñar, de lo que quieres que aprenda y tienes que hacer un proceso de sistematización entre lo que quieres que aprendan y el proyecto. Simplemente tú les presentas una situación problemática y el alumno tiene que resolverla de acuerdo a sus habilidades.

Gestiona: El profesor se dedica a guiar, conducir, controlar tiempos, dar pautas, redirigir objetivos, supervisar las actividades y productos del alumno y en caso de ser necesario corregir las posibles estrategias de solución en los proyectos (Tippelt y Lindemann, 2001).

PG: [Mi labor consiste en] aprobar el proyecto. Conforme se van viendo los temas les indico qué es lo que tienen que ir haciendo y reviso los instrumentos antes de que los apliquen.

Evalúa: Además de valorar las producciones generadas por los estudiantes, el profesor también analiza los resultados globales obtenidos en el grupo para que, en caso de ser necesario, actualice o rediseñe el proyecto para su próxima implementación.

PE: Nos enfrentamos a la evaluación de cambio de actitud. En mejoras en las actitudes y al final tuvimos que improvisar y buscar un instrumento que nos pudiera ayudar a ver ese cambio. Precisamente cuando yo lo implementé no estaba muy desarrollado el estudio de actitudes [ni] de instrumentos para evaluar actitudes, pero es algo que consideramos que debe incluirse.

[Con respecto al proyecto] el profesor [a veces tiene que] abordar temas que al principio no estaban considerados, y al final, precisamente, la exigencia del mundo real obliga a ir adecuándose a lo que los alumnos necesitan.

\section{Categoría 3: Sobre el papel del Alumno}

En esta categoría se consideran las funciones que el profesor asigna a los estudiantes de manera voluntaria o involuntaria. Basados en la literatura especializada y en los datos 
DOI: https://doi.org/10.20396/zet.v26i3.8650908

recabados, dividimos a esta categoría en las subcategorías Elige el tema, Produce, Expone los resultados/productos, No participante.

Elige el tema: El profesor considera que los alumnos deben elegir el tema del proyecto que de acuerdo a su interés sea factible de investigar y realizar (Richit y Vinicius, 2010).

PG: Los estudiantes escogen el tema, motivados porque no se les impone.

Produce: El alumno es responsable de buscar respuestas a determinadas cuestiones (Tippelt y Lindemann, 2001). Dentro de esta producción, investiga, organiza, participa activamente en una o en cada una de las actividades del proyecto.

PE: En el proyecto [los alumnos] hacen los cálculos y además las actividades de andamiaje que dejas en el aula cuando se dan los temas. Resuelven problemas y todo el desarrollo [del proyecto].

Expone los resultados o los productos: El alumno comunica a sus compañeros y al profesor de manera oral o escrita los resultados obtenidos en la investigación, o los productos desarrollados en el proyecto.

PA: Un proyecto es aquel en el que entregas un trabajo terminado. Uno por semestre. El examen final es el proyecto. Al final del curso entregan todo su banco de trabajos.

No participante: Pese a que en la literatura de investigación en ABP no se aborda esta posibilidad, hemos decidido incluir esta categoría para contemplar dicha posibilidad, ya que el instrumento está diseñado para explorar la comprensión de los profesores y tener estudiantes no participativos puede pertenecer (consciente o inconscientemente) a su comprensión sobre el ABP. Nos referimos a los casos en donde el alumno se limita sólo a reproducir un método predeterminado por el profesor. Tampoco tenemos evidencias para ilustrar esta categoría.

\section{Categoría 4: Sobre los aspectos que favorecen o afectan la incorporación del ABP como metodología de trabajo.}

En esta categoría se consideran los aspectos inherentes al profesor de matemáticas o al alumno, así como los vinculados a la institución, que pudieran afectar o beneficiar la implementación del ABP en el aula. Los subelementos de esta categoría son: Disponibilidad, infraestructura, coordinación con otros profesores, experiencia del docente y experiencia del alumno.

Disponibilidad: Se refiere a la actitud de renuencia o aceptación de los estudiantes frente al proyecto (Tippelt y Lindemann, 2001).

PA: [Se requiere] disponibilidad de los estudiantes, porque sin ello no se logra nada. Al principio [su actitud] es de rechazo porque es más trabajo, creo que a nadie nos gusta que nos den más trabajo. Posteriormente cambia mucho, hasta se 
DOI: https://doi.org/10.20396/zet.v26i3.8650908

sienten satisfechos de que pueden hacer cosas que al principio suenan muy difíciles.

Infraestructura: Se considera como el lugar, instalaciones y recursos para realizar el proyecto.

PE: La técnica que hemos aplicado con profesores y alumnos, es una experiencia impresionante, porque simplemente sacarlos del aula y llevarlos al contexto en el caso del puente [se refiere a un proyecto que realizaron analizando la construcción de un paso peatonal elevado], cambia la visión de la gente.

PA: El proyecto se puede ver afectado por la falta de un lugar donde hacerlo.

Coordinación con otros profesores: Describimos a esta actividad como la disposición e interés del profesor para colaborar con otros profesores, ya sea de matemáticas o de otras asignaturas, ante la gestión de los proyectos en la materia.

PA: En bachillerato me es más difícil, porque no les doy a todos los grados, el estudio es más de área. A ellos los mandé a hacer algunos proyectos como medir la casa del alfeñique [se refiere a un edificio de valor cultural en Puebla, México, lo cual relaciona con proyectos que mezclan capacidades y conocimientos de otras áreas de conocimiento distintas a las matemáticas]. Otra cosa que se me ocurre es trabajar las matemáticas en la vista [y así analizar] el ojo humano.

Experiencia Docente: Se piensa en este campo como aquellos aspectos que se han adquirido durante la experiencia como docentes, ya sea trabajando con el método de ABP o con cualquier otra metodología, pero que repercutan en la implementación actual del ABP.

PA: La ventaja son los años de experiencia, cuando eres recién egresada tienes lo de tus estudios universitarios y lo que crees que se puede usar, y con tantos años he experimentado una cosa, otra y he visto que unas cosas funcionan con determinadas personas, lo que funciona este año, puede ser que el siguiente no.

PE: Si el profesor nunca ha hecho investigación, no va a poder dar lo que no tiene. Lo típico de los profesores de la asignatura de Metodología que enseñan, pero nunca han escrito un artículo. El profesor mismo ya debió haber tenido esa experiencia de trabajar con aprendizaje basado en proyectos. [Por otra parte] cuando un profesor ya ha dado muchos años una forma de dar clase es muy difícil sacarlo de esa sinergia que tiene.

Experiencia del alumno: Se supone como la característica que posee el alumno por haber trabajado con anterioridad este método o en circunstancias acordes al mismo. También se toman en cuenta las consideraciones que realiza el profesor respecto a cómo influye la experiencia del estudiante con metodologías distintas de enseñanza (incluidas las de corte tradicionalista). 
DOI: https://doi.org/10.20396/zet.v26i3.8650908

PE: Es impresionante ver cómo, según la perspectiva [del alumno] y su formación, van a dar soluciones a los problemas, entonces los problemas reales tienen muchas soluciones. Y cuando todos están proponiendo sus soluciones, les permite ver la realidad desde una perspectiva más compleja, menos limitada. El aprendizaje colaborativo que se logra es impresionante. Los chicos aprenden mucho más, no sólo el conocimiento [sino también] habilidades qué tienen que ver con el contexto social en la reflexión, el compromiso con el entorno, con tus compañeros, hasta saber negociar, se adquieren habilidades de argumentación, habilidades para redactar un proyecto, para hacer un cartel.

PA: [Suelo notar] falta de coordinación entre ellos, porque es trabajo en equipos y luego no se pueden coordinar para ciertas actividades. No saben escuchar, no saben seguir instrucciones.

\section{Categoría 5: Sobre evaluación}

Nos referimos en esta categoría al proceso que realiza el profesor para medir el avance de los alumnos. Teniendo en cuenta su conocimiento matemático, habilidades, disponibilidad o esfuerzo y sobre el sistema que utiliza para recoger los datos del proyecto desde la propuesta, ejecución y presentación del mismo.

Tipos: Con este término nos referimos a los objetivos de la evaluación diagnostica, sumativa o formativa. Así como al enfoque metodológico cuantitativo, cualitativo o mixto. Y al agente evaluador (autoevaluación, co-evaluación o heteroevaluación).

PE: Con respecto a la evaluación obviamente tienes que valorar ciertos contenidos de tu tema, pero en el prototipo [del proyecto] te das cuenta [qué aprendieron]. En el proyecto te hacen los cálculos, las actividades que dejas en el aula, se les enseña ciertas habilidades con las cuales también se evalúan. [Se evalúa] la presentación y todo el desarrollo. Algunas evaluaciones son individuales y otras son colectivas, depende la actividad que se les ponga. [La evaluación] de actitudes son individuales, las exposiciones orales se evalúan individualmente, aparte está el examen.

Instrumentos: Nos referimos a todas aquellas herramientas que le sirven al profesor como apoyo para la evaluación del desempeño del alumno como lo son la lista de cotejo, rubrica, portafolio de evidencia u otros.

PG: [para la evaluación del proyecto] nos apoyamos por rubricas en las distintas etapas.

Momentos: Describimos este aspecto como el tiempo en el que el profesor somete a evaluación los productos obtenidos del proyecto. Los cuales pueden ser al inicio del proyecto, durante todo el proyecto, o al término de este. También nos da cuenta del papel del proyecto en la evaluación general de los estudiantes. 
DOI: https://doi.org/10.20396/zet.v26i3.8650908

PA: El curso lo evaluó por medio de trabajos, diario casi hago examen, es un trabajo continuo, la evaluación es continua, no puedes decir si me haces el examen bien, ya tienes 10', el examen final es el proyecto.

\section{Categoría 6: Aspectos que condicionan la elección, diseño y gestión del proyecto}

Esta categoría cuenta con subelementos que pueden ser determinantes en la elección, diseño y gestión del proyecto, así como de la decisión del profesor de matemáticas de implementar la metodología de Aprendizaje Basado en Proyectos en su centro de trabajo.

Programa: El programa al que se adscribe la institución donde labora el profesor puede ser un obstáculo o un aliciente para el desarrollo del proyecto.

PA: A partir del control [de los avances que pide el programa académico] uno pone el proyecto y no al revés, primero el programa, avances, mejoras de los estudiantes y luego un proyecto que les beneficie a ellos.

Tiempos: Con este subelemento pretendemos conocer el tiempo mínimo y máximo que los profesores consideran apropiado para realizar un proyecto y también si el trabajo con el proyecto puede interferir con el cumplimiento de las metas del programa educativo en cada sección del curso.

PE: El proyecto es una integración de los temas vistos con lo que no tengo problema de atrasarme.

PG: Necesitas tiempo para que los alumnos puedan procesar la información. Podríamos pensar en mínimo un mes, dos meses si se lleva a cabo más tiempo. A lo largo del cuatrimestre estaría ideal.

Temas que no trabajaría con ABP: Nos referimos a aquellos temas o conceptos que el profesor no considera apropiado/posible trabajar bajo el método de Aprendizaje basado en Proyectos y las razones que tiene para pensarlo así.

PE: Ahora mismo estoy con un joven de maestría [los estudiantes de esa maestría son profesores en activo]. Él trabajó reducción de fracciones algebraicas, y aquí el reto sería: ¿cómo aplicas ese diseño en ABP? Entonces ese sería un reto para el profesor [se refiere a ese mismo estudiante, pero ahora lo visualiza en su rol como profesor], porque hay temas bastante abstractos, un poco áridos. Por ejemplo, si vas a dar teoría de la medida, llega un momento en que todo está más como en la imaginación. Pero bachillerato se presta mucho más para implementar esas estrategias. Creo que depende de la imaginación del diseñador. A lo mejor no existe límite, nada más es necesario que el diseñador pueda lograr el vínculo, porque hay teorías matemáticas que todavía no tienen aplicación en la vida real.

De esta manera, se conformó el instrumento que se muestra en la Figura 2, el cual, además de proporcionar una categorización que permite analizar distintas facetas del posible 
pensamiento del profesor, también muestra una variedad exhaustiva sobre las formas en las que podría comprender dichos elementos.

\begin{tabular}{|c|c|}
\hline $\begin{array}{c}\text { Elección del problema o } \\
\text { temática }\end{array}$ & $\begin{array}{l}\text { - Real } \\
\text { - Cuasi- Real } \\
\text { - Ficticios } \\
\text { - Puro }\end{array}$ \\
\hline Papel del Profesor & $\begin{array}{l}\text { - Diseña } \\
\text { - Gestiona } \\
\text { - Evalúa }\end{array}$ \\
\hline Papel del Alumno & $\begin{array}{l}\text { - Elige el tema } \\
\text { - Produce } \\
\text { - Expone los resultados o productos } \\
\text { - No participante }\end{array}$ \\
\hline $\begin{array}{l}\text { Aspectos que favorecen o afectan } \\
\text { la incorporación del ABP como } \\
\text { metodología de trabajo }\end{array}$ & $\begin{array}{l}\text { - Disponibilidad } \\
\text { - Infraestructura } \\
\text { - Coordinación con otros } \\
\text { - Experiencia Docente /Experiencia alumno }\end{array}$ \\
\hline Evaluación & $\begin{array}{l}\text { - Tipos } \\
\text { - Instrumentos } \\
\text { - Momentos }\end{array}$ \\
\hline $\begin{array}{l}\text { Aspectos que condicionan } \\
\text { la elección, diseño y } \\
\text { gestión del proyecto }\end{array}$ & $\begin{array}{l}\text { - Programa } \\
\text { - Tiempos } \\
\text { - Temas que no trabajarían con ABP }\end{array}$ \\
\hline
\end{tabular}

Figura 2. Instrumento resultante para explorar la caracterización que hacen los profesores de matemáticas acerca del Aprendizaje Basado en Proyectos

\section{Conclusiones}

Esta investigación, de corte cualitativo, nos proporcionó un instrumento para explorar la caracterización que realizan profesores acerca del uso de la metodología denominada Aprendizaje Basado en Proyectos. Con esto, se puede interpretar qué es lo que entienden dichos profesores de matemáticas sobre la definición y aplicación del ABP. Esta interpretación nos permite visualizar que el profesor ha creado su teoría personal acerca del $\mathrm{ABP}$, proveniente, en algunos casos, de teorías formales, y en otros de la práctica en el aula.

Constatamos lo que se dice en Perreneoud (2000), que cada profesor tiene una relación personal con los proyectos en el aula, que muy probablemente tiene que ver con su experiencia en la docencia y en la vida personal. Algunos son movidos a implementar esta metodología por la creencia de que el puro conocimiento matemático es poco motivador si no se sabe de su utilidad real (Batanero y Díaz, 2004). En estos casos incorporan a su currículo proyectos para apoyar lo que se ve en clase. Algunos otros, en concordancia con Tippelt y Lindemann (2001) toman como eje principal el proyecto complementándolo con otros métodos de aprendizaje que lo faciliten y lo van adecuando para dirigir al estudiante al descubrimiento y adquisición del conocimiento matemático en turno.

En lo que pudimos observar y que planteamos en nuestro análisis, todos determinan la elección y gestión del proyecto dependiendo también de otros factores como los 
institucionales o los inherentes a los alumnos. Pudimos constatar que los profesores también se ven limitados por la dificultad que les da vincular ciertos contenidos matemáticos a un proyecto en el contexto del alumno.

En la construcción de la caracterización teórica del ABP encontramos un amplio bagaje de proyectos, pero según lo que notamos en las entrevistas, los profesores no se apoyan en estos proyectos que ya han sido validados, pese a que ello significaría un ahorro en el trabajo y planeación, además de la posibilidad de contrastar sus resultados con los de las investigaciones. Esto nos da oportunidad, en un futuro, de explorar los efectos que pueda tener que los profesores lleven a sus aulas dichos proyectos.

En esta investigación aportamos tres resultados principales: el primero se refiere a una caracterización, proveniente de la literatura de investigación, en términos de propiedades, fortalezas y debilidades del ABP. Cabe señalar que las debilidades reportadas tienen un fundamento en experiencias de profesores que han trabajado con el método y que algunas de estas aparecieron en nuestro análisis. Sin embargo, notamos que la conceptualización que realiza el profesor que trabaja con ABP suele ser más en términos positivos, aunque no siempre concordante con la caracterización realizada en la literatura.

El segundo aporte es una entrevista semiestructurada elaborada con base en la caracterización teórica y es un instrumento inédito para la recogida de datos en los procesos investigativos en los que el caso de estudio es un profesor de matemáticas que ha trabajado con ABP. Este instrumento es conveniente para investigaciones de corte cualitativo en donde se explore la imagen mental que ha formado el profesor acerca de la metodología. También puede utilizarse como prueba diagnóstica en cursos de actualización sobre ABP.

El tercer resultado principal, el cual consideramos que es más relevante, es la determinación de categorías y subcategorías que puede servir como instrumento de observación para evaluar el trabajo del docente de matemáticas con el ABP. Nosotros lo hemos construido con base en la evidencia de entrevistas, pero también con sustento en literatura de investigación sobre ABP y sobre temas que se relacionan directamente con la categoría en cuestión. Con ello intentamos crear un espectro amplio que permita considerar diversas comprensiones que tenga el profesor.

Las investigaciones que estudiamos para elaborar este artículo abordan las implicaciones, en términos de motivación y aprendizajes, que tiene en el estudiante el trabajo con ABP. Pero no encontramos investigaciones que se centraran en el profesor (e.g. conocimientos que requiere, concepciones predominantes, toma de decisiones). Así, consideramos que hemos contribuido a explorar un campo en el que se ha indagado poco.

Como se sabe en los estudios de corte social, como es el nuestro, los resultados no son totalmente generalizables, el paradigma de investigación provee resultados situados. Esto significa que, aunque es una caracterización valida porque está respaldada por la literatura especializada y un estudio empírico detallado, puede ser mejorada si aportamos más casos de estudio, puesto que se esperaría confirmar lo encontrado aquí o en el mejor de los casos 
DOI: https://doi.org/10.20396/zet.v26i3.8650908

ampliar los elementos de la caracterización del ABP que no figuren en este estudio.

\section{Agradecimientos}

Esta investigación se realizó gracias al financiamiento del Consejo Nacional de Ciencia y Tecnología otorgado mediante la Beca No. CVU 738065 y de la Secretaría de Educación Pública de México mediante el programa PRODEP.

\section{Referencias}

Anijovich, R., \& Mora, S. (2010). Estrategias de enseñanza: otra mirada al quehacer en el aula. Buenos Aires: Aique Grupo Editor.

Aravena, M. (2002). Las principales dificultades en el trabajo algebraico. Un estudio con alumnos de ingeniería de la UCM. Revista Académica UC Maule, 28, 63-81.

Aravena, M., Caamaño, C., \& Giménez, J. (2008). Modelos matemáticos a través de proyectos. Revista Latinoamericana de Investigación en Matemática Educativa RELIME, 11(1), 49-92

Batanero, C., \& Díaz, C. (2004). El papel de los proyectos en la enseñanza y aprendizaje de la estadística. En J. Patricio (Ed.), Aspectos didácticos de las matemáticas (pp.125-164). Zaragoza: ICE.

Benjumeda, F.J., Romero, I., \& López-Martín, M. (2015). Alfabetización matemática a través del aprendizaje basado en proyectos en secundaria (Eds.), Investigación en Educación Matemática XIX (pp. 163-172). Alicante: SEIEM..

Blasco T., \& Otero, L. (2008) Técnicas conversacionales para la recogida de datos en investigación cualitativa: La entrevista (II). Nure Investigación, 34, 1-6.

Carrillo, J., \& Muñoz-Catalán, M.C. (2011). Análisis metodológico de las actas de la SEIEM (1997-2010) desde la perspectiva de los métodos cualitativos. Reflexión en torno a un caso. En M. Marín, G. Fernández, L. Blanco, \& M. Palarea (Eds.), Memorias del XV Simposio de la Sociedad Española de Investigación en Educación Matemática (pp. 99116). Ciudad Real, España: SEIEM.

Espinosa-Mirabet, S., Soler, M., Escoda, M., Puig-Bargués, J., \& Ferrer, I. (2015). Un modelo para diseñar aprendizajes mediante proyectos multidisciplinares. REDU -Revista de Docencia Universitaria, 13(3), 73-88.

Falcao, V. (2014). Resolución de problemas de matemáticas en Educación Primaria: influencia del contexto. Tesis de maestría inédita. Cantabría: Universidad de Cantabría, España.

Galeana, L. (2006). Aprendizaje basado en proyectos. Revista CEUPROMED, 1(27), 1-17.

Morales, L., \& García O. (2015). Un aprendizaje basado en proyecto en matemática con alumnos de undécimo grado. Números, 90, 21-30.

Morales, C. (2011). El Aprendizaje basado en Proyectos en la Educación Matemática del siglo XXI, Cuaderno de bitácora. $15^{\circ}$ JAEM Jornadas sobre el Aprendizaje y Enseñanza de las matemáticas (pp. 1-23) Canarias - España: JAEM. 
DOI: https://doi.org/10.20396/zet.v26i3.8650908

Moreti, M.T. (2009). O jogo das relações didáticas sob a influência dos projetos de trabalho The didatical relations games with the influency of the works projects, Zetetiké: Revista de Educação Matemática, 11(20), 110-139.

Nestingen, M. (2002). Reading Your Way Into Culture: A Materials Development Project. Master's thesis. School for International Training, Brattleboro, Vermont.

Niss, M. (2006). The concept and role of theory in mathematics education. En C. Bergsten, B. Grevholm, H. Måsøval y F. Rønning (Eds.), Relating Practice and Research in Mathematics Education. Proceedings of Norma 05 (pp. 97-110). Trondheim, Noruega.

Perrenoud, P. (2000). Aprender en la Escuela a través de Proyectos: ¿Por qué?, ¿Cómo? Revista de Tecnología Educativa, XIV(3), 311-321.

Tippelt, R., \& Lindemann, H. (2001). El método de proyectos. El Salvador: Ministerio de Educación.

Thomas, J.W. (2000). A review of research on project-based learning. San Rafael-California,

CA: Autodesk Foundation.

Reverte, J., Gallego, A., Molina, R., \& Satorre, R. (2007) El aprendizaje basado en proyectos como modelo docente. Experiencia interdisciplinar y herramientas groupware, Actas de las XIII Jornadas de Enseñanza Universitaria de la Informática. S.D.

Richit, A., \& Vinicius, M. (2010). Desafios e Possibilidades do Trabalho com Projetos e com Tecnologias na Licenciatura em Matemática, Zetetiké, 18(33), 15-41.

Skovsmose, O. (2001). Landscapes of investigation. ZDM, 33(4), 123-132.

Valero, M. (2007). L'aprenentatge basat en projectes en els ensenyaments tècnics. Perspectiva Escolar, 318, 42-50.

Valles, M. (2002). Entrevistas cualitativas. (Colección Cuadernos metodológicos, num. 32). Madrid: CIS.

Villa-Ochoa, J., Rojas, C., \& Cuartas, C. (2010). ¿Realidad en las matemáticas escolares?: Reflexiones acerca de la "realidad" en modelación en educación matemática. Revista Virtual Universidad Católica del Norte, 29, 1-17

Wiest, L. (2001). The role of fantasy contexts in word problems. Mathematics Education Research Journal, 13(2), 74-90. 